\title{
Deflection of Structures Using Principle of Quasi Work
}

\author{
Inder Kris hen Panditta ", M. Maruf Wani
}

Mechanical Engineering Department, N .I. T. Srinagar; Hazratbal, J\&K, 190006, India

\begin{abstract}
A new methodology for obtaining deflections of structures is presented in this paper. It is based on Principle of Quasi Work, which connects two topologically similar systems thereby leading to a unique concept of standard elements for every category of structural problems. Using a priory known equation of deformation/deflection of elastic axis of these elements, solution for equation of deformed/ deflected elastic line of other structural members of similar category having different loading and boundary conditions is presented here. Th is methodology is easy to use as deflection of a given structure is obtained mostly by simple multiplications and it also eliminates the use of internal force/bending moment expressions unlike conventional methods. Even though this methodology can be applied to any structure, its use is illustrated for one dimensional structural elements (viz. axial bars, torsion rods and beams) for the sake of concis eness and clarity. The concept of topologically similar system is exp lored for each category of elements as it lies at the heart of the principle of quasi work.
\end{abstract}

Keywo rds Energy Methods, Structural Analysis, Beams, PQW, TSS, TSB, TEB

\section{Introduction}

Success ful attempts of transporting structural mechanics problems into other fields of science have been made by various authors. Structural mechanics problems were solved using graph theory by[1, 2]; through electrical networks by[3]; and resorting to combination of graph theory and electric networks by[4]. In all these cases, one needs knowledge of other fields of science in addition to the knowledge of structural mechanics. To overcome this crippling disadvantage Principle of Quasi Work (PQW) in the realm of structural mechanics was derived by [5] by importing Tellegen's theorem as given in[6] thereby making knowledge of other fields of science for any user redundant. PQW establishes connection between two Topologically Similar Systems, (TSS), in the field of structural mechanics.All the presently available structural analysis procedures e.g. finite element methods $[7,8,9,10]$; variational principles $[11,12]$ and energy methods [13, 14] meant for a single structure do not provide any connection between two structural systems. PQW thus fills existing void in the domain of structural mechanics. Using PQW, some useful theorems for discrete structural models were derived by [5, 15]. PQW was advantageously used by [16] for obtaining redundant reactions of beams. A quick and simplified method for obtaining nodal deflections of an indeterminate truss using PQW was given by[17]. PQW has much wider applicability as all the existing energy principles

* Corresponding author:

ikpandita@rediffmail.com (Inder Krishen Panditta)

Published online at http://journal.sapub.org/aerospace

Copyright (C) 2012 Scientific \& Academic Publishing.All Rights Reserved can be derived as special cases of PQW by restricting TSS to topologically identical systems i.e. when two systems are identical with each other in every respect.PQW utilizes known solution of a TSS to solve other determinate/ indeterminate topologically similar problems.

All the available methods for calculation of deflection are based on internal force (/ torque/ bending moment/ etc.) distributions. PQW gives a choice of either using internal force expressions or completely bypassing these. First case (of using these distributions) is a direct application of PQW $\left(\mathrm{W}_{\mathrm{mn}}=\mathrm{U}_{\mathrm{mn}}\right.$ ). In the latter case (of bypassing these distributions), solution is obtained by restricting TSS to topologically equivalent systems (TES). For TES $\mathrm{U}_{\mathrm{mn}}=\mathrm{U}_{\mathrm{nm}}$ and hence, PQW can be used in the form of $\mathrm{W}_{\mathrm{mn}}=\mathrm{W}_{\mathrm{nm}}$. Working with quasi work expressions is much easier compared to quasi strain energy expressions. Quasi work $\left(\mathrm{W}_{\mathrm{mn}}\right)$ is calculated by multiplying applied loads from system ' $\mathrm{m}$ ' with corresponding known displacements of system ' $n$ ' and no integrations are involved if only concentrated loads are acting on the given system. Even for distributed loads the integration is easy as the integrants (the expression for distributed load acting on the given system, $\mathrm{TES}_{\mathrm{m}}$, and the equation for the deflection curve of chosen system $\mathrm{TES}_{\mathrm{n}}$ ) are predefined. PQW involves two TSS out of which one represents the given determinate/ indeterminate problem at hand and otherTSS with a priory known equation of its deformed/ deflected elastic line is chosen by the user.Hence, a judicious choice of $\mathrm{TSS}_{2}$ leads to a unique standard element for every category of problem.

Deformation/ deflection equations of these standard elements, used for obtaining the deformation/ deflection of other given determinate/ indeterminate problems (of the same class) under different loading and boundary conditions, 
remain same thereby paving way for development of a general purpose interactive computer program for obtaining the deflection of these structural members.

In this paper, conditions which axial bars and torsion rods have to satisfy for topological similarity are looked into. PQW is validated for these elements. An attempt is made to define standard element (in each case) whose solution for deflection/ deformation forms the basis for obtaining deflections/ deformations of other given problems. Few illustrative examples are also included.

\section{Principle of Quasi Work}

Principle of quasi work was first proved by Panditta[5] and subsequently stated by Panditta et.al.[15,16]. According to this principle quasi work, ' $\mathrm{W}_{\mathrm{mn}}$ ', done by external forces of one system (say ' $m$ ') while going through the corresponding displacements of the other topologically similar system (say ' $n$ ') is equal to the quasi strain energy, ' $U_{m}$ ', due to internal forces of former system (' $\mathrm{m}$ ') while going through corresponding deformations of the latter system ('n'). In mathematical terms it can be stated as:

$$
W_{m n}=U_{m n}
$$

Where, $\mathrm{U}_{\mathrm{mn}}=$ Quasi strain energy $=\{F\}_{m}^{T}\{\delta\}_{n} ; \mathrm{W}_{\mathrm{mn}}=$ Quasi work $=\{P\}_{m}^{T}\{d\}_{n} ;\{\mathrm{P}\}_{\mathrm{m}}=$ External loads acting on $\mathrm{TSS}_{\mathrm{m}}\{\mathrm{d}\}_{\mathrm{n}}=$ Displacement in $\mathrm{TSS}_{\mathrm{n}}$ corresponding to $\{\mathrm{P}\}_{\mathrm{m}}$; $\{\mathrm{F}\}_{\mathrm{m}}=$ Internal forces in $\mathrm{TSS}_{\mathrm{m}} ;\{\delta\}_{\mathrm{n}}=$ Deformation in $\mathrm{TSS}_{\mathrm{n}}$ corresponding to $\{\mathrm{F}\}_{\mathrm{m}}$ and $\{(\exp .)\}_{\mathrm{i}}=$ (expr.) is for $\mathrm{TSS}_{\mathrm{i}}$.

In this principle, word 'corresponding' needs clarification. For example, take two beams 'AB' and ' $\mathrm{CD}$ ' having lengths ' $L_{1}$ ' and ' $L_{2}$ '. In these two beams end ' $A$ ' corresponds to end ' $\mathrm{C}$ ' and end ' $\mathrm{B}$ ' to end ' $\mathrm{D}$ '. In such cases, one has to resort to mapping in the two structural domains. For example, if a transverse load is acting at $0.75 \mathrm{~L}_{1}$ fromend ' $\mathrm{A}$ ' in beam ' $\mathrm{AB}$ ' then its corresponding point in beam 'CD' will be $0.75 \mathrm{~L}_{2}$ from end ' $\mathrm{C}$ '.

\subsection{Topol ogical Similarity of Structural Elements}

In this section, condition(s) which axial bars and torsion rods should satisfy for being topologically similar will be arrived at. These conditions are deduced from calculations of quasi energy associated with the two structural elements. This also sets the conditions for the applicability of PQW as quasi energy is a part of PQW.

\subsubsection{Topological Similar A xial Bars}

Let two axial bars with parameters $\mathrm{L}_{\mathrm{m}}, \mathrm{E}_{\mathrm{m}}, \mathrm{A}_{\mathrm{m}}$ and $\mathrm{L}_{\mathrm{n}}, \mathrm{E}_{\mathrm{n}}$, $\mathrm{A}_{\mathrm{n}}$ be represented by $\mathrm{TSS}_{\mathrm{m}}$ and $\mathrm{TSS}_{\mathrm{n}}$, respectively. Quasi energy, ' $\mathrm{U}_{\mathrm{mn}}$ ' is written as follows:

$$
U_{m n}=\int_{A_{m}} \int_{0}^{L_{n}}\left\{\sigma_{m}\left(L_{n} x_{m} / L_{m}\right) d A_{m}\right\}_{m}\left\{\varepsilon_{n}\left(x_{n}\right) d x_{n}\right\}_{n}
$$

Where, $\sigma(\mathrm{x})=$ Normal stress as a function of ' $\mathrm{x}$ '; $\varepsilon(\mathrm{x})=$
Normal Strain as a function of ' $\mathrm{x}$ '; $\mathrm{A}=$ Cross section area; $\mathrm{L}=$ Length of structural member; $\mathrm{m}, \mathrm{n}=$ Subscripts representing different TSS and ' $x$ ' is the coordinate along longitudinal axis.

As length of the two bars is different, mapping of $x$-coordinate from TSS $_{m}$ to TSS $S_{n}$ gives $x_{m} L_{n} / L_{m}$ and $x_{n}$ as corresponding points in these TSS. In such a situation it is better to work in terms of non- dimensional parameter $\xi=x_{m}$ $/ L_{m}=x_{n} / L_{n}$. Eqn.(2) in non-dimensional variable becomes:

$$
\begin{aligned}
U_{m n} & =\int_{A_{m}} \int_{0}^{1}\left\{\sigma_{m}\left(L_{n} \xi\right) d A_{m}\right\}_{m}\left\{\varepsilon_{n}\left(L_{n} \xi\right) L_{n} d \xi\right\}_{n} \\
& =\int_{A_{m}}^{1} \int_{0}^{1}\left\{\frac{F_{m}\left(L_{n} \xi\right)}{A_{m}} d A_{m}\right\}_{m}\left\{\frac{F_{n}\left(L_{n} \xi\right)}{A_{n} E_{n}} L_{n} d \xi\right\}_{n} \\
& =\int_{0}^{1} \frac{L_{n}}{A_{n} E_{n}} F_{m}\left(L_{n} \xi\right) F_{n}\left(L_{n} \xi\right) d \xi
\end{aligned}
$$

Similarly

$$
U_{n m}=\int_{0}^{1} \frac{L_{m}}{A_{m} E_{m}} F_{m}\left(L_{m} \xi\right) F_{n}\left(L_{m} \xi\right) d \xi
$$

Where, $\mathrm{E}=$ Young's modulus of elasticity. If mapping is done from $\mathrm{TSS}_{\mathrm{n}}$ to $\mathrm{TSS}_{\mathrm{m}}$ then quasi energy is given by:

$$
\begin{aligned}
U_{m n} & =\int_{0}^{1} \frac{L_{m}}{A_{n} E_{n}} F_{m}\left(L_{m} \xi\right) F_{n}\left(L_{m} \xi\right) d \xi \text { and } \\
U_{n m} & =\int_{0}^{1} \frac{L_{n}}{A_{m} E_{m}} F_{m}\left(L_{n} \xi\right) F_{n}\left(L_{n} \xi\right) d \xi
\end{aligned}
$$

For deriving Eqn.(3) no restrictions on any geometric parameters of the two bars was required. Hence, two bars having different geometric parameters are topologically similar. It paves way for the application of $\mathrm{PQW}$. If topologically similar axial bars (TSAB) also have same distribution of the parameter $\mathrm{AE}\left(\mathrm{A}_{\mathrm{m}} \mathrm{E}_{\mathrm{m}}=\mathrm{A}_{\mathrm{n}} \mathrm{E}_{\mathrm{n}}\right)$ along their length then these axial elements will be designated as Topologically Equivalent Axial Bars (TEAB). For these elements quasi strain energy $U_{m n}$ given by Eqn.(3), simplifies to:

$$
U_{m n}=\int_{0}^{1} \frac{L_{n}}{A E} F_{m}\left(L_{n} \xi\right) F_{n}\left(L_{n} \xi\right) d \xi
$$

For TEAB, Eqns. (4 and 5) give $\mathrm{U}_{\mathrm{mn}}=\mathrm{U}_{\mathrm{nm}}$ and along with Eqn. (1) offer six equations (viz. $\mathrm{W}_{\mathrm{mn}}=\mathrm{U}_{\mathrm{mn}}=\mathrm{U}_{\mathrm{nm}}=\mathrm{W}_{\mathrm{nm}}$ ) for their analysis instead of usual two equations $\mathrm{W}_{\mathrm{mm}}=\mathrm{U}_{\mathrm{mn}}$ and $\mathrm{W}_{\mathrm{nm}}=\mathrm{U}_{\mathrm{nm}}$. If the length of the TEAB is also equal then the Eqn.(5) is written as:

$$
U_{m n}=\int_{0}^{L} \frac{F_{m}(x) F_{n}(x)}{A E} d x
$$

Illustration - 1:Validation for A xial Bars

Consider two axial bars with parameters $\mathrm{A}_{1}, \mathrm{E}_{1}, \mathrm{~L}_{1}$ and 
$\mathrm{A}_{2}, \mathrm{E}_{2}, \mathrm{~L}_{2}$. Let the ends of first member be fixed - fixed (Fig.1a) and that of second be fixed - free (Fig. 1b).

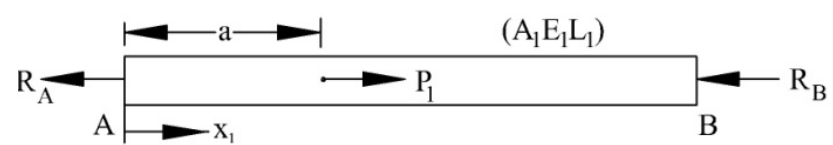

a) Fixed - Fixed bar: $\mathrm{TSAB}_{1}$

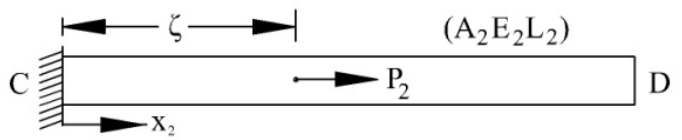

b) Built-in Bar/Standard bar: $\mathrm{TSAB}_{2} / \mathrm{TEAB}_{2}$

Figure 1. Topologically Similar Bars

Loading on first be a force ' $\mathrm{P}_{1}$ ' Newton acting at a distance of ' $\mathrm{a}$ ' meters and that of the other be ' $\mathrm{P}_{2}$ ' Newton acting at a distance of ' $\zeta$ ' meters measured from left end in both the cases. Let $R_{A}$ and $R_{B}$ be the support reactions. First one will be designated by $\mathrm{TSAB}_{1}$ and other by $\mathrm{TSAB}_{2}$. Internal fo rce distribution, $\mathrm{F}(\xi)$, and axial deformation, $\mathrm{u}(\xi)$, in terms of non-dimensional parameter ' $\xi$ ' for $\mathrm{TSAB}_{1}$ and $\mathrm{TSAB}_{2}$ are given hereunder:

$$
\begin{aligned}
& \left\{F_{1}(\xi)\right\}_{1}=R_{A}-P_{1}<\xi-a / L_{1}>^{0} \\
& \left\{u_{1}(\xi)\right\}_{1}=L_{1}\left(R_{A} \xi-P_{1}<\xi-a / L_{1}>\right) / A_{1} E_{1} \\
& \left\{F_{2}(\xi)\right\}_{2}=P_{2}-P_{2}<\xi-\zeta / L_{2}>^{0} \\
& \left\{u_{2}(\xi)\right\}_{2}=L_{2} P_{2}\left(\xi-<\xi-\zeta / L_{2}>\right) / A_{2} E_{2}
\end{aligned}
$$

Where, $<$ (exp.) $>=0$ if (exp.) $\leq 0$ otherwise it is equal to (exp.). Using Eqn.(3)

$$
\begin{aligned}
U_{12}= & \left\{-R_{B} \zeta+P_{1}(\zeta-<\zeta-a>\}_{1}\left\{P_{2} / A_{2} E_{2}\right\}_{2}\right. \\
\text { and } & \\
W_{12}= & \left\{-R_{A}\right\}_{1}\left\{u_{2}(0)\right\}_{2}+\left\{P_{1}\right\}_{1}\left\{u_{2}\left(a / L_{1}\right)\right\}_{2} \\
& +\left\{-R_{B}\right\}_{1}\left\{u_{2}(1)\right\}_{2} \\
= & 0+\left[\left\{P_{1}\right\}_{1}\left\{a / L_{1}-<a / L_{1}-\zeta / L_{2}>\right\}_{2}\right. \\
& \left.-\left\{R_{B}\right\}_{1}\left\{\left(1-<1-\zeta / L_{2}>\right)\right\}_{2}\right]\left\{P_{2} L_{2} / A_{2} E_{2}\right\}_{2} \\
= & {\left[-\left\{R_{B}\right\}_{1}\{\zeta\}_{2}+\left\{P_{1}\right\}_{1}\left\{\zeta-<\zeta-a L_{2} / L_{1}>\right\}_{2}\right] } \\
& \left\{P_{2} / A_{2} E_{2}\right\}_{2}
\end{aligned}
$$

Referring displacements to TSAE $E_{1}$, Eqn.(10) can be written as Eqn.(11) or Eqn.(12).

$$
\begin{aligned}
& W_{12}=\left\{-R_{B} \zeta+P_{1}(\zeta-<\zeta-a>)\right\}_{1}\left\{P_{2} / A_{2} E_{2}\right\}_{2} \\
& W_{12}=\left\{-R_{B} \zeta+P_{1}(a-<a-\zeta>)\right\}_{1}\left\{P_{2} / A_{2} E_{2}\right\}_{2}
\end{aligned}
$$

From Eqns. $(9,11)$ PQW stands validated.Similarly one can show that $\mathrm{U}_{21}=\mathrm{W}_{21}$.

\subsubsection{Topological Similar Shafts}

Representing two shafts with circular cross section and having parameters $\mathrm{L}_{\mathrm{m}}, \mathrm{G}_{\mathrm{m}}, \mathrm{J}_{\mathrm{m}}$ and $\mathrm{L}_{\mathrm{n}}, \mathrm{G}_{\mathrm{n}}, \mathrm{J}_{\mathrm{n}}$ by $\mathrm{TSS}_{\mathrm{m}}$ and $\mathrm{TSS}_{\mathrm{n}}$, the expression for $\mathrm{U}_{\mathrm{mn}}$ derived on similar lines as for axial elements is given below:
$U_{m n}=\int_{A_{m}} \int_{0}^{L_{n}}\left\{\tau_{m}\left(L_{n} x_{m} / L_{m}\right) d A_{m}\right\}_{m}\left\{\gamma_{n}\left(x_{n}\right) d x_{n}\right\}_{n}$

In non-dimensional form, $\mathrm{U}_{\mathrm{m}}$ is given by:

$$
\begin{aligned}
& U_{m n}=\int_{A_{m}} \int_{0}^{1}\left\{\tau_{m}\left(L_{n} \xi\right) d A_{m}\right\}_{m}\left\{\gamma_{n}\left(L_{n} \xi\right) L_{n} d \xi\right\}_{n} \\
& =\int_{A_{m}} \int_{0}^{1}\left\{\frac{T_{m}\left(L_{n} \xi\right) r_{m}}{J_{m}} d A_{m}\right\}_{m}\left\{\frac{T_{n}\left(L_{n} \xi\right) r_{n}}{J_{n} G_{n}} L_{n} d \xi\right\}_{n} \\
& =\int_{0}^{1} L_{n}\left\{\frac{T_{m}\left(L_{n} \xi\right)}{J_{m}}\right\}_{m}\left\{\frac{T_{n}\left(L_{n} \xi\right)}{G_{n} J_{n}}\right\}_{n} d \xi \int_{A_{m}} r_{m} r_{n} d A_{m}
\end{aligned}
$$

Where, $\tau(x)=$ Shear stress as a function of ' $x$ '; $\gamma(x)=$ Shear strain as a function of ' $\mathrm{x}$ '; $\mathrm{J}=$ Polar moment of cross sectional area; $G=$ Shear modulus of elasticity and $T(x)$ is applied torque as a function of $\mathrm{x}$.

For meaningful evaluation of the integral over $A_{m}$, it will be prudent to take two shafts of equal diameter. With this corresponding points in radial direction on the two shafts will be at the same radial distance from the shaft centres (i.e. $\left.\mathrm{r}_{\mathrm{m}}=\mathrm{r}_{\mathrm{n}}\right)$ and $\mathrm{J}_{\mathrm{m}}=\mathrm{J}_{\mathrm{n}}(=\mathrm{J})$. The second integral evaluates to $\mathrm{J}_{\mathrm{m}}$ $(=J)$.With this, expression for $\mathrm{U}_{\mathrm{m}}$ in Eqn. (14) reduces to:

$$
U_{m n}=\int_{0}^{1} \frac{L_{n}}{G_{n} J} T_{m}\left(L_{n} \xi\right) T_{n}\left(L_{n} \xi\right) d \xi
$$

If mapping is done from $\mathrm{TSS}_{\mathrm{n}}$ to $\mathrm{TSS}_{\mathrm{m}}$, then $\mathrm{U}_{\mathrm{mn}}$ takes the form:

$$
U_{m n}=\int_{0}^{1} \frac{L_{m}}{G_{n} J} T_{m}\left(L_{m} \xi\right) T_{n}\left(L_{m} \xi\right) d \xi
$$

Derivation of Eqn. (15) necessitates that the radius of two shafts should be equal. Hence, for shafts to be topologically similar their diameter should be equal. For topologically equivalent rods (TER) with $\mathrm{G}_{\mathrm{m}}=\mathrm{G}_{\mathrm{n}}=\mathrm{G}$ Eqn. $(15,16)$ gives $\left(\mathrm{W}_{\mathrm{mn}}=\right) \mathrm{U}_{\mathrm{mn}}=\mathrm{U}_{\mathrm{nm}}\left(=\mathrm{W}_{\mathrm{nm}}\right)$.

\section{Illustration - 2: Validation for Shafts}

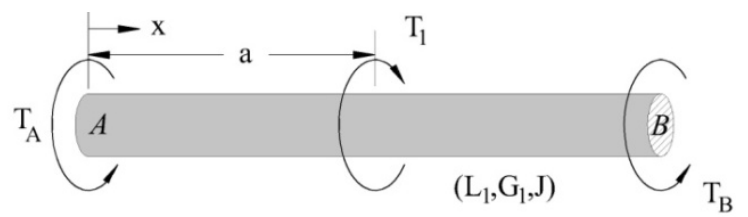

a) Fixed - Fixed Rod: TSR

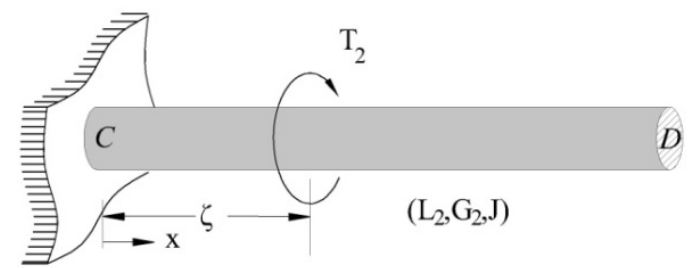

b) Built-in Rod/Standard Rod: $\mathrm{TSR}_{2} / \mathrm{TER}_{2}$

Figure 2. Topologically Similar Rods 
Consider a fixed - fixed torsion rod with circular section and having parameters $\mathrm{J}, \mathrm{G}_{1}, \mathrm{~L}_{1}$ and an anticlockwise torque ' $\mathrm{T}_{1}$ ' $\mathrm{Nm}$ acting at a distance of ' $\mathrm{a}$ ' meters from left end ' $A$ ' as shown in (Fig. 2a). This rod will be designated by $\mathrm{TSS}_{1}$. Topologically similar rod, $\mathrm{TSS}_{2}$, will be taken as a fixed - free torsion rod with circular section having parameters $\mathrm{J}, \mathrm{G}_{2}, \mathrm{~L}_{2}$ and an anti-clockwise torque ' $\mathrm{T}_{2}$ ' N.m acting at a distance of ' $\zeta$ ' meters fro $m$ left end ' $C$ ' as shown in (Fig. 2b).

The non - dimensional internal torque, $\mathrm{T}(\mathrm{x})$, and angle of twist, $\theta(\mathrm{x})$, distributions in $\mathrm{TSR}_{1}$ and TSR 2 a re given by:

$$
\begin{aligned}
& \left\{T_{1}(\xi)\right\}=T_{A}-T_{1}<\xi-a / L_{1}>^{0} \\
& \left\{\theta_{1}(\xi)\right\}=L_{1}\left(T_{A} \xi-T_{1}<\xi-a / L_{1}>\right) / G_{1} J \text { and } \\
& \left\{T_{2}(\xi)\right\}=T_{2}-T_{2}<\xi-\zeta / L_{2}>^{0} \\
& \left\{\theta_{2}(\xi)\right\}=L_{2}\left(T_{2} \xi-T_{2}<\xi-\zeta / L_{2}>\right) / G_{2} J \\
& \text { and } \mathrm{W}_{12} \text { are given by: } \\
& \qquad U_{12}=T_{2}\left[-T_{B} \zeta+T_{1}(\zeta-<\zeta-a>] / G_{2} J\right. \\
& \quad W_{12}=T_{2}\left[-T_{B} \zeta+T_{1}(\zeta-<\zeta-a) / G_{2} J\right.
\end{aligned}
$$

$\mathrm{U}_{12}$ and $\mathrm{W}_{12}$ are given by:

From Eqns.(18,19) PQW stands validated. Same is true for $\mathrm{U}_{21}$ and $\mathrm{W}_{21}$.

\subsubsection{Topological Similar Beams}

Condition for topologically similar beams has been derived by Panditta et.al.[16] and it requires that the two beams should have equal depth. Quasi strain energy, $U_{m n}$, of two topologically similar beams (TSB) with parameters (L, $\left.A_{m}, E_{m}\right)$ and $\left(L, A_{n}, E_{n}\right)$, referred to as $\mathrm{TSB}_{m}$ and $\mathrm{TSB}_{n}$, is given by:

$$
U_{m n}=\int_{0}^{L} \frac{1}{E_{n} I_{n}}\left\{M_{m}(x)\right\}_{m}\left\{M_{n}(x)\right\}_{n} d x
$$

For topologically equivalent beams (TEB) with $\mathrm{E}_{\mathrm{m}} \mathrm{I}_{\mathrm{m}}=\mathrm{E}_{\mathrm{n}}$ $\mathrm{I}_{\mathrm{n}}=$ EI, Eqn. (20) along with Eqn. (1) as usual yield six equations for their analysis.

\section{Standard Elements}

After validating PQW for one dimensional elements it can be observed that PQW relates two structural systems through quasi wo $\mathrm{k}$ and quasi strain energy. Hence, it is possible to use known solution of appropriately predefined $\mathrm{TSS}_{2}$ to get solution of all other determinate/ indeterminate problems $\left(\mathrm{TSS}_{1}\right)$ of the same class. In other words, one can utilize expression for internal force (for calculating $U_{m n}$ ) and deformation/ deflections (for obtaining $\mathrm{W}_{\mathrm{mn}}$ ) of one unique TSS (of a class) and utilize it for obtaining solutions of other TSS (of the same class). This is of particular interest in structural engineering as one has to deal with indeterminate structures whose solution can now be obtained by the solution of a determinate structure (predefined $\mathrm{TSS}_{2}$ ) of the same class. Moreover, there is a possibility of utilizing exclusively quasi work expressions (which do not need internal force distribution) by choosing two systems to be
TES which necessitates thatthe two structural systems should have same stiffness parameter (AE/ GJ/ EI/ etc.). Here an attempt is made to define standard elements for axial bars, torsion rods and beam elements. These elements will be referred to as standard elements.

Axial member shown in Fig. 1b will be used as a standard bar. It will be designated as $\mathrm{TSAB}_{2} / \mathrm{TEAB}_{2}$. Cantilever shaft with circular section shown in Fig. $2 \mathrm{~b}$ will be used as a standard torsion rod. It will be designated as $\mathrm{TSR}_{2} / \mathrm{TER}_{2}$.

Standard beam for deflection (SBD), $\mathrm{TSB}_{2} / \mathrm{TEB}_{2}$, is chosen as a simply supported beam ' $C D$ ' with parameters $\mathrm{L}$, $E_{2}, I_{2}$ as shown in Fig.3. Load on the beam is taken as a concentrated load ' $\mathrm{P}_{2}$ ' Newton acting at a distance of ' $\zeta$ ', meters from left end ' $C$ '. Equation for its deflected neutral axis is given by:

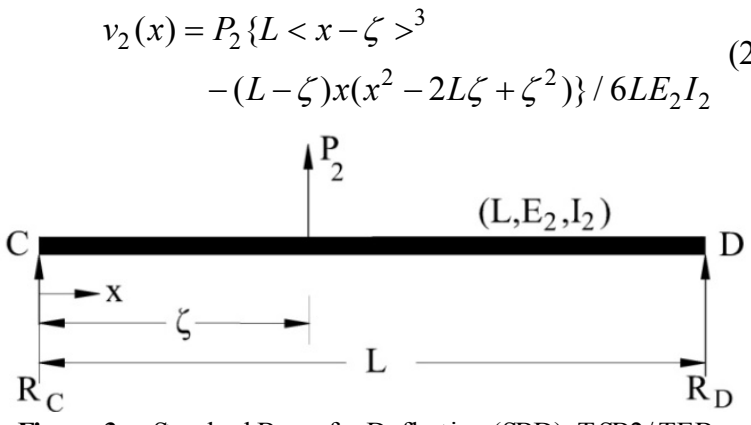

Figure 3. Standard Beam for Deflection (SBD): $\mathrm{T} \mathrm{SB} 2 / \mathrm{TEB}_{2}$

The beam, shown in Fig.(4), will be taken as standard beam for slope (SBS). It is defined as a simply supported beam ' $\mathrm{CD}$ ' with an anticlockwise moment ' $\mathrm{M}_{2}$ ' acting at a distance ' $\zeta$ ' from the left end. The beam parameters are taken as E,I, L. SBS will be designated as $\mathrm{TSB}_{2} / \mathrm{TEB}_{2}$ as the situation demands. This SBS can be used for getting slope of the deflected neutral axis of given beams. Equation of its deflected elastic curve is given by:

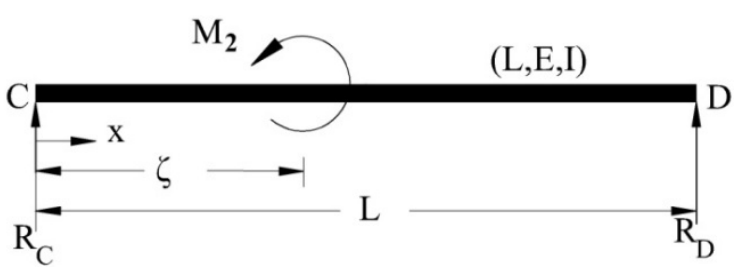

Figure 4. Standard Beam for Slope (SBS): $\mathrm{T} \mathrm{SB}_{2} / \mathrm{TEB}_{2}$

$$
\begin{aligned}
v_{2}(x)= & M_{2}\left\{x\left(x^{2}+2 L^{2}+3 \zeta^{2}-6 L \zeta\right)\right. \\
& \left.-3 L<x-\zeta>^{2}\right\} / 6 L E I
\end{aligned}
$$

\section{Deflection Using PQW}

In this section five illustrations are included to illustrate the use of PQW for axial bar, torsion rod and beams. In all these illustrations two systems are chosen as TES of each other and having same length. By opting for TES it becomes easy as one can use quasi work expressions which make internal force/ torque/ bending moment expressions redundant. As these expressions are not needed, one need not 
have the knowledge of writing these expressions thereby making the process of obtaining deflections easier and faster. Two illustrations one each for axial bar and shaft, even though trivial, are included to impart clarity in understanding of concepts involved.

\subsection{Application to Axial B ars}

In this section equation for deformation of fixed - fixed bar given in Fig.(1a) is obtained by using the equation of deformation of a built-in bar given in Fig( $1 b)$. As it is easy to obtain quasi work, these bars willbe chosen as TEAB having same parameters L, A and E.

Eqn.(12) with modified parameters yields:

$$
\begin{gathered}
W_{12}=\left\{P_{2} / A E\right\}_{2}\left\{\left[-R_{B} \zeta+P_{1}(a-<a-\zeta>)\right]\right\}_{1} \\
\text { And } W_{21}=\left\{P_{2}\right\}_{2}\left\{u_{1}(\zeta)\right\}_{1}
\end{gathered}
$$

Using PQW in the form $\mathrm{W}_{21}=\mathrm{W}_{12}$ yields:

$$
\left\{u_{1}(\zeta)\right\}_{1}=\left\{\left[-R_{B} \zeta+P_{1}(a-<a-\zeta>)\right] / A E\right\}_{1}
$$

$\mathrm{R}_{\mathrm{B}}$ is calcu lated by using the boundary condition that $\mathrm{u}_{1}(\mathrm{~L})$ $=0$ and comes out to be equal to $\mathrm{P}_{1} \mathrm{a} / \mathrm{L}$. Substituting this value of $R_{B}$ in the above equation, the expression for axial deformation of the given fixed - fixed axial bar becomes:

$$
\left\{u_{1}(\zeta)\right\}_{1}=\left\{P_{1}[a(1-\zeta / L)-<a-\zeta>] / A E\right\}_{1}
$$

As $\zeta$ is an arbitrary point in $\mathrm{TEAB}_{2}$, it can as well be replaced by ' $\mathrm{x}$ ' and if curly brackets are also dropped then Eqn. (26) takes conventional form.

\subsection{Application to Rods}

In this section PQW will be applied to a fixed - fixed shaft, shown in Fig. 5, to obtain its equation of twist by making use of the equation of twist of the standard rod shown in Fig.2b.

The given shaft in Fig.5 carries a distributed torque of $t^{\prime}$ ' $\mathrm{N}$. $\mathrm{m} / \mathrm{m}$ and has parameters $\mathrm{G}, \mathrm{J}, \mathrm{L}$. Let $\mathrm{T}_{\mathrm{A}}$ and $\mathrm{T}_{\mathrm{B}}$ be the reaction torques in anticlockwise direction acting at left support 'A' and right support ' $B$ ', respectively. This shaft will be designated as $\mathrm{TER}_{1}$.

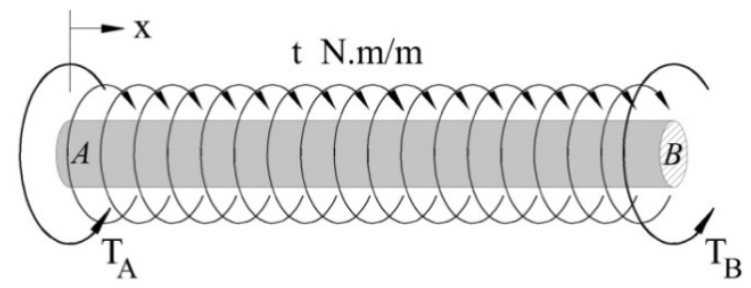

Figure 5. Fixed - Fixed Shaft: TER

Standard torsion rod shown in Fig. $2 \mathrm{~b}$ will be chosen to have same parameters $\mathrm{G}, \mathrm{J}, \mathrm{L}$ so that it is topologically equivalent to the given shaft and will be referred to as $\mathrm{TER}_{2}$. Equation for twist as a function of ' $\mathrm{x}$ ' for $\mathrm{TER}_{2}$ is given by:

$$
\left\{\theta_{2}(x)\right\}_{2}=\left[T_{2} x-T_{2}<x-\zeta>\right] / G J
$$

$\mathrm{W}_{12}$ is calculated as follows:

$$
\begin{aligned}
W_{12} & =\left\{T_{A}\right\}_{1}\{\theta(0)\}_{2}+\int_{0}^{L}\{-t\}_{1}\{\theta(x)\}_{2}+\left\{T_{B}\right\}_{1}\{\theta(L)\}_{2} \\
& =\left(\left\{-T_{2} t\left[x^{2}-<x-\zeta>^{2}\right]_{0}^{L}\right\} / 2+T_{2} T_{B} \zeta\right) / G J \\
& \left.=\left\{T_{2}\right\}_{2}\left\{\zeta\left[t(\zeta / 2-L)+T_{B}\right)\right] / G J\right\}_{1}
\end{aligned}
$$

$$
\text { And } W_{21}=\left\{T_{2}\right\}_{2}\left\{\theta_{1}(\zeta)\right\}_{1}
$$

Using PQW in the form $\mathrm{W}_{21}=\mathrm{W}_{12}$ yields:

$$
\left\{\theta_{1}(\zeta)\right\}_{1}=\zeta\left[T_{B}+t(\zeta / 2-L)\right] / G J
$$

$\mathrm{T}_{\mathrm{B}}$ is obtained to be equal to $\mathrm{tL} / 2$ by applying the boundary condition $\theta(\mathrm{L})=0$. Substituting this value in Eqn.(30), expression for twist of fixed-fixed shaft becomes:

$$
\left\{\theta_{1}(\zeta)\right\}_{1}=t \zeta(\zeta-L) / 2 G J
$$

As $\zeta$ is an arbitrary point in $\mathrm{TER}_{2}$ it is also an arbitrary point in the given problem $\left(\mathrm{TER}_{1}\right)$ which has same length as that of $\mathrm{TER}_{2}$, hence, represents the same location as in $\left(\mathrm{TER}_{1}\right)$. Therefore, if $\zeta$ is rep laced by ' $\mathrm{x}$ ' and curly brackets are dropped, Eqn.(31) will take the conventional form.

\subsection{Application to Beams}

In this section PQW will be applied to beams. Three beam problems are chosen for this purpose. First and second problem is for obtaining the expression for deflection of a determinate and an indeterminate beam, respectively. Last one demonstrates the use of PQW for getting the expression for slope of a determinate beam.

Illustration - 3: Deflection of Determinate Beam

$\mathrm{TEB}_{1}$ represents the given problem which in this illustration is chosen as a simply supported beam 'AB' of length ' $L$ ' with an overhang of length ' $a$ ' to the left of the support ' $A$ ' as shown in Fig.6. The loading on it is taken as an anticlockwise moment $M_{1}$ acting at the free end and a uniform load of intensity $\mathrm{w}_{1} \mathrm{~N} / \mathrm{m}$ between the supports. Let its parameters be $\mathrm{E}$ and I (second moment of area). It is required to find the equation for the deflected neutral axis between the supports. SBD (Fig.3) is chosen as $\mathrm{TEB}_{2}$ having same parameters E, I and L.

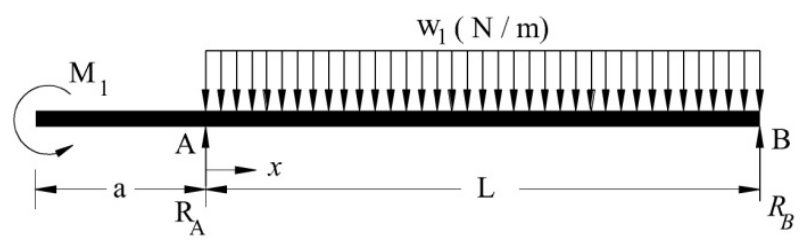

Figure 6. Beam with overhang: $\mathrm{TEB}_{1}$

As the equation for neutral axis between the supports is required, the end moment $M_{1}$ is statically transferred to support ' $\mathrm{A}$ '. $\mathrm{W}_{12}$ is calculated as follows:

$$
\begin{aligned}
W_{12}= & \left\{M_{1}\right\}_{1}\left\{v_{2}^{\prime}(0)\right\}_{2}+\int_{0}^{L}\left\{-w_{1}\right\}_{1}\left\{v_{2}(x)\right\}_{2} d x \\
& +\left\{R_{A}\right\}_{1}\left\{v_{2}(0)\right\}_{2}+\left\{R_{B}\right\}_{1}\left\{v_{2}(L)\right\}_{2} \\
= & -\left(P_{2} / E I\right)\left[M_{1}(L-\zeta)\left(\zeta^{2}-2 L \zeta\right) / 6 L\right. \\
& \left.+w_{1}(L-\zeta)\left(L^{2} \zeta+L \zeta^{2}-\zeta^{3}\right) / 24\right] \\
& W_{21}=\left\{P_{2}\right\}_{2}\left\{v_{1}(\zeta)\right\}_{1}
\end{aligned}
$$

As the two beams were chosen to be TES of each other, deflection at any point $\zeta$ in $\mathrm{TEB}_{1}$ is obtained by using PQW in the form $\mathrm{W}_{21}=\mathrm{W}_{12}$ and comes out to be:

$$
\begin{aligned}
\left\{v_{1}(\zeta)\right\}_{1}= & -(L-\zeta)\left[w_{1}\left(L^{2} \zeta+L \zeta^{2}-\zeta^{3}\right)\right. \\
& \left.+4 M_{1}\left(\zeta^{2}-2 L \zeta\right) / L\right] / 24 E I
\end{aligned}
$$


If $\mathrm{M}_{1}=\mathrm{w}_{1} \mathrm{~L}^{2} / 12$ then Eqn.(34) simp lifies to:

$$
\left\{v_{1}(\zeta)\right\}_{1}=-w_{1}\left(3 \zeta^{4}-7 L \zeta^{3}+3 L^{2} \zeta^{2}+L^{3} \zeta\right) / 72 E I
$$

Replacing $\zeta$ by $\mathrm{x}$ and dropping suffix ' 1 ' as well as curly brackets in the above equation yields the equation for deflection in conventional form which is same as given in[18]

Illustration - 4: Deflection of Indeterminate Beam

In this illustration equation for the deflected neutral axis of a beam $A B$ which has a roller support at the left end ' $A$ ' and is built-in at support ' $\mathrm{B}$ ' is obtained. Beam has parameters $\mathrm{L}$, $\mathrm{E}$, I and carries an antic lockwise mo ment ' $\mathrm{M}$ ' at its mid point as shown in Fig. 7 wherein $R_{A}, R_{B}$ and $M_{B}$ are reactions from supports. This beam is designated as $\mathrm{TEB}_{1}$. SBD (Fig.3) is chosen as $\mathrm{TEB}_{2}$.

Quasi work $\mathrm{W}_{12}$ is given by:

$$
\begin{aligned}
W_{12}=P_{2}\left[M \left\{3 L<L / 2-\zeta>^{2}\right.\right. & \\
& \left.-(L-\zeta)\left(3 L^{2} / 4+\zeta^{2}-2 L \zeta\right)\right\} \\
& \left.-M_{B}(L-\zeta) \zeta(L+\zeta)\right] / 6 E I L \text { and } \\
W_{21}= & P_{2} v_{1}(\zeta)
\end{aligned}
$$

Using $\mathrm{W}_{21}=\mathrm{W}_{12}, \mathrm{v}_{1}(\xi)$ comes out to be equal to:

$$
\begin{aligned}
v_{1}(\zeta)= & {\left[M \left\{3 L<L / 2-\zeta>^{2}\right.\right.} \\
& \left.-(L-\zeta)\left(3 L^{2} / 4+\zeta^{2}-2 L \zeta\right)\right\} \\
& \left.-M_{B}(L-\zeta) \zeta(L+\zeta)\right] / 6 E I L
\end{aligned}
$$

Using boundary condition that slope at $\zeta=\mathrm{L}$ is zero, y ields value of $M_{B}$ as $M / 8$. With this value of $M_{B}$ the equation for the deflection becomes:

$$
\begin{aligned}
v_{1}(\zeta)= & M[24 L<L / 2-\zeta\rangle^{2} \\
& -8(L-\zeta)\left(3 L^{2} / 4+\zeta^{2}-2 L \zeta\right) \\
& -(L-\zeta) \zeta(L+\zeta)] / 48 E I L
\end{aligned}
$$

This on simplification y ields:

$$
v_{1}(\zeta)=M\left[9 \zeta^{3} / L-3 L \zeta+24<\zeta-L / 2>^{2}\right] / 48 E I \text { (39) }
$$

Replacing $\zeta$ by $x$ and substituting $2 \mathrm{~L}$ for $\mathrm{L}$ y ields the same equation as given in[18]

Illustration - 5: Slope of Determinate Beam

Problem given in illustration -3 is here taken up again for obtaining the equation of the slope of the neutral axis. $\mathrm{TEB}_{2}$ is chosen as SBS (Fig.4) with equation for deflection given by Eqn.(22). Quasi work, $\mathrm{W}_{12}$, is calculated as follows:

$$
\begin{aligned}
W_{12}= & \left\{M_{1}\right\}_{1}\left\{v_{2}^{\prime}(0)\right\}_{2}+\int_{0}^{L}\left\{-w_{1}\right\}_{1}\{v(x)\}_{2} d x \\
& +\left\{R_{A}\right\}_{1}\left\{v_{2}(0)\right\}_{2}+\left\{R_{B}\right\}_{1}\left\{v_{2}(L)\right\}_{2} \\
= & M_{2}\left[12 M_{1}\left(2 L^{2}+3 \zeta^{2}-6 L \zeta\right) / L\right. \\
& \left.-w_{1}\left(3 L^{3}-18 L \zeta^{2}+12 \zeta^{3}\right)\right] / 72 E I \text { and } \\
W_{12}= & M_{2} v_{1}^{\prime}(\zeta)
\end{aligned}
$$

Using $\mathrm{W}_{21}=\mathrm{W}_{12}$, expression for slope is given by:

$$
\begin{aligned}
v_{1}^{\prime}(\zeta)= & {\left[12 M_{1}\left(2 L^{2}+3 \zeta^{2}-6 L \zeta\right) / L\right.} \\
& \left.-w_{1}\left(3 L^{3}-18 L \zeta^{2}+12 \zeta^{3}\right)\right] / 72 E I
\end{aligned}
$$

If $\mathrm{M}_{1}$ is taken to be equal to $\mathrm{w}_{1} \mathrm{~L}^{2} / 12$, then the above equation reduces to:

$$
v_{1}^{\prime}(\zeta)=-w_{1}\left(12 \zeta^{3}-21 L \zeta^{2}+6 L^{2} \zeta+L^{3}\right) / 72 E I
$$

As expected above equation is the derivative of Eqn.(35)

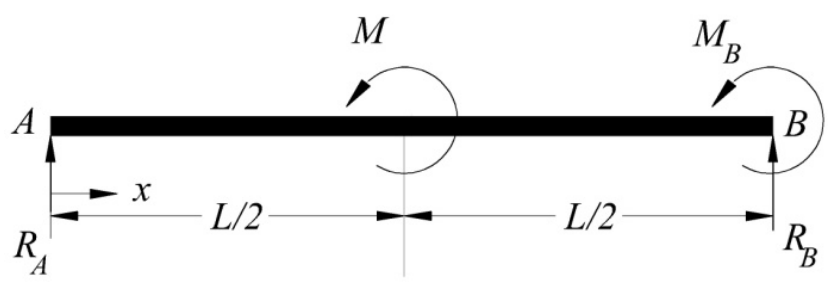

Figure 7. Indeterminate Beam: $\mathrm{TEB}_{1}$

\section{Conclusions}

1). PQW can be applied to a set of any two axial bars.

2). PQW is applicable to a pair of rods/ shafts so long as these have same radius of cross section.

3). A xial, torsion and beam standard elements are defined. With the help of the equation of deformed/ deflected elastic line of these predefined standard ele ments and PQW solution for deflection of other problems of the same class is obtained with ease.

4). Obtaining deflection/deformations at a point or equation of deflected/deformed elastic line of given problems using PQW is further simplified if the given problem and its corresponding standard element are chosen to be topologically equivalent. This is due to:

i ) The knowledge of internal force (/torque/bending mo ment) is not required.

ii ) For distributed loads one definite integral is to be evaluated whose integrant is the product of two a priory known expressions (viz. distributed load of the given problem and the equation of deflected elastic line of its standard element).

iii) For point loads it is simpler and easier as even the integration is not required. For this kind of loading,sum of multip lications of known loads of the given problem with known deflections/ deformations at the corresponding points in the chosen topologically similar system is all that is required.

5). As solutions of standard elements (for deflection/ deformation of its elastic line) are used to solve any other problem of the same class with different loads and different constraints, it is possible to develop an interactive graphic general purpose computer package for this purpose.

6). The concepts of virtual force and complementary energy do not exist in PQW as both the systems are real. Hence, no additional effort is needed for learn ing.

7). $\mathrm{PQW}$ is used for solving indeterminate problems with the help of the solution of their determinate topologically similar system.

\section{REFERENCES}

[1] N., Ta'aseh, O. and Shai, "Graph theoretical duality 
perspective on conjugate structures and its applications", European Journal of Mechanics A/Solids, vol. 24, 2005, pp $974-986$.

[2] Fenves, S.J. and Branin, F.H., "Network Topological Formulations of Structural Analysis", Journal of Structural Division, ASCE, vol. 89, 1963, pp 483 - 514.

[3] Sebastian Wendel M., "Collapse Considerations and Electrical Analogies for Statically Indeterminate Structures", Journal of Structural Engineering, ASCE, vol. 130, 2004, pp $1445-1453$.

[4] Shai, O., "Deriving Structural Theorems and Methods using Tellegen's Theorem and Combinatorial Representations", International Journal of Solids and Structures, vol. 38, 2001, pp $8037-8052$.

[5] Panditta, I.K., "Some Studies on Computer Aided Model Based Design”, Ph. D. Dissertation, IIT Bombay, India, 1996.

[6] Penfield, P. Jr., Spencer, R. and Duinker, S., “Tellegen's Theorem and Electrical Networks", Research Monograph No.58, The M.I.T. Press, Cambridge, 1970, pp $1-24$.

[7] Akin, J.E., "Finite Elements for Analysis and Design", Academic Press, London, 1994.

[8] Cook, R.D., Malkus, D.J. and Plesha, M.E., "Concepts and Applications of Finite Element Analysis”, John Wiley \& sons, New York, 1989.

[9] Zienkiewicz, O. C. and Taylor, R. L., "The Finite Elements Method", McGraw Hill Book Co., 1989.
[10] Rao, S. S., "The Finite Element Method in Engineering", Pergamon Press, 1989.

[11] Reissner, E., "Formulations of Variational Theorems in Geometrically Non-linear Elasticity" Journal of Engineering Mechanics, Vol. 110, No. 9, 1984, pp 1377 - 1390.

[12] Reissner, E., "On Mixed Variational Formulations in Finite Elasticity”, Acta Mech., Vo. 56, No. 3-4, 1985, pp 117-125.

[13] Argyris, J. H. and Kelsey, S., "Energy Theorems and Structural Analy sis”, Butterworth \& Co. Ltd., 1960.

[14] Shames, I.A. and Dym, C.L., "Energy and Finite Element methods in Structural Mechanics", McGraw - Hill Book Co., 1985.

[15] Panditta, I.K., Shimpi, R.P. and Prasad, K.S.R.K., "On the Theory of Discrete Model Analyses and Design", International Journal of Solids and Structures, Vol. 36, 1999, pp 2443-2462.

[16] Panditta, I.K., Ambardhar, R. and Dembi, N.J., "Redundant Reactions of Indeterminate Beams by Principle of Quasi Work", AIAA journal, DOI: 10.2514/1.42470.

[17] InderKrishen Pandita, "Derivative Theorems of the Principle of Quasi Work", International Journal of Aerospace Sciences, DOI: $10.5923 /$ j.aerospace. 20120103.02

[18] Riley, W.F., Strurges, D. and Morris, D.H., "Mechanics of Materials", fifth edition, John Wiley \& Sons, (Asia) Pte. Ltd., Singapore, pp 480 and 524. 